\title{
Unusual Evolution of Bilateral Neuroblastoma
}

\section{Jamila $\mathrm{E}^{1 *}$, Mohamed $\mathrm{O}^{2}$, Mohamed $\mathrm{S}^{3}$ and Mhamed $\mathrm{H}^{1}$}

1Pediatric hematology and Oncology unit, University Hospital Mohammed VI, Marrakesh, Morocco

2Pediatric surgery B, Medical school of Marrakesh, Cady Ayad University, Morocco

${ }^{3}$ Pediatric department B, Medical school of Marrakesh, Cady Ayad University, Morocco

\section{Case Report}

Volume 2 Issue 3

Received Date: June 01, 2018

Published Date: June 15, 2018

${ }^{*}$ Corresponding author: Jamila Elhoudzi, Pediatric hematology and Oncology unit, University Hospital Mohammed VIMarrakesh, Morocco, Email: jelhoudzi@gmail.com

\section{Abstract}

Introduction: Bilateral neuroblastoma is extremely rare. To date, only 60 cases are reported in the literature. The association of neuroblastoma with paraneoplastic Cushing's syndrome is exceptional. The average age at diagnosis is 3 months. The majority of patients are classified according to INSS stage IVs. The prognosis is generally favorable with $90 \%$ survival at 5 years. The treatment is similar to unilateral neuroblastoma.

Case Report: 3 months girl, admitted for excessive weight gain with bluish skin nodules. These symptoms started on the 40th day of life. There were no gastrointestinal symptoms, nor exophthalmia, obesity with a weight of $7.7 \mathrm{~kg}$, the face puffy with telangiectasias, arterial hypertension $(15 / 08 \mathrm{cmHg})$ and hepatomegaly at $6 \mathrm{~cm}$ below the costal margin. Abdominal ultrasound showed a right adrenal mass of $7.7 / 9 \mathrm{~cm}$ heterogeneous structure--second adrenal mass was found on the left side with the same characteristics. The liver is nodular and increased size. The rest of the radiological assessment was normal (thorax and skeleton).

The bone marrow smear was normal. The level of cortisol was high at $8 \mathrm{pm}$, urinary catecholamine were normals, and biopsy of subcutaous nodules showed a malignant tumor proliferation suggesting at first around cell neuroblastoma or PNET. Research NMYC amplification was not done. The diagnosis of bilateral neuroblastoma with liver metastasis associated with Cushing's syndroma is retained. We had proceeded with resection at the left side and partial at the right one. The child is placed on antihypertensive therapy and ultrasound monitoring. The short-term trend showed a decline in blood pressure with decreased volume of subcutaneous nodules and weight. After 8 months, the patient has presented a cerebral metastasis and died.

Conclusion: The bilateral nature and association with Cushing's syndrome does not seem to have a negative impact on prognosis neuroblastoma. Frequently reported spontaneous regression in neuroblastoma stage IVs is observed in the case reported here; but the cerebral complication wasn't expected in such cases.

Keywords: Bilateral neuroblastoma; Cushing's syndrome; Brain metastasis 


\section{Case Report}

Nada was born on May 29/2009. She was admitted to our unit at the age 3 months for excessive weight gain with bluish skin nodules. The onset of symptoms started since the 40th day of life. There were no gastrointestinal symptoms and no exophthalmia. Obesity was striking with a weight of $7.7 \mathrm{~kg}$ (over 3DS), while birth weight was $2 \mathrm{~kg}$, her face puffy with telangiectasias. Arterial hypertension at 15/08cmHg and hepatomegaly at $6 \mathrm{~cm}$ below the costal margin were noticed. Bluish nodules were on the right supraclavicular level, right side, right axillary, bilateral breast, the right buttock at the edge of the right hand side and pubis, but no peripheral lymph nodes or exophthalmia were found (Figure 1).

Abdominal ultrasound and CT scan showed a right adrenal mass of 7.7 by $9 \mathrm{~cm}$ heterogeneous structure displacing down the kidney and the liver forward. There was another adrenal mass on the left side to $3 / 4 \mathrm{~cm}$ with the same characteristics. The liver was nodular and increased size (Figure2). The rest of the radiological assessment was normal (chest X Ray and skeleton). The bone marrow smear was normal. The level of cortisol in blood was high at $08 \mathrm{~h} 560 \mathrm{ng} / \mathrm{ml}(\mathrm{N}=50$ à 110$)$, urinary catecholamines were normals, and biopsy of subcutaneous nodules showed a malignant tumor proliferation suggestive of malignant round tumor cells probably neuroblastoma or PNET.

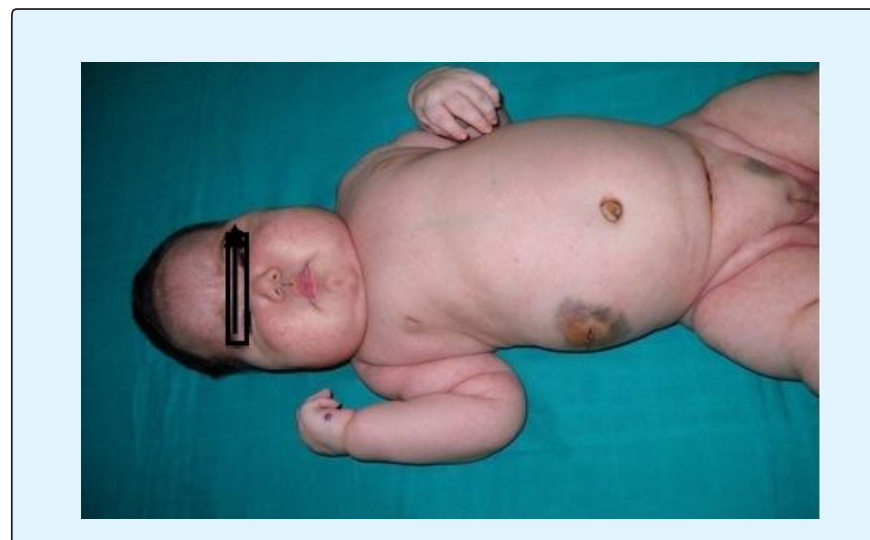

Figure 1: Cutaneous nodules at diagnosis.
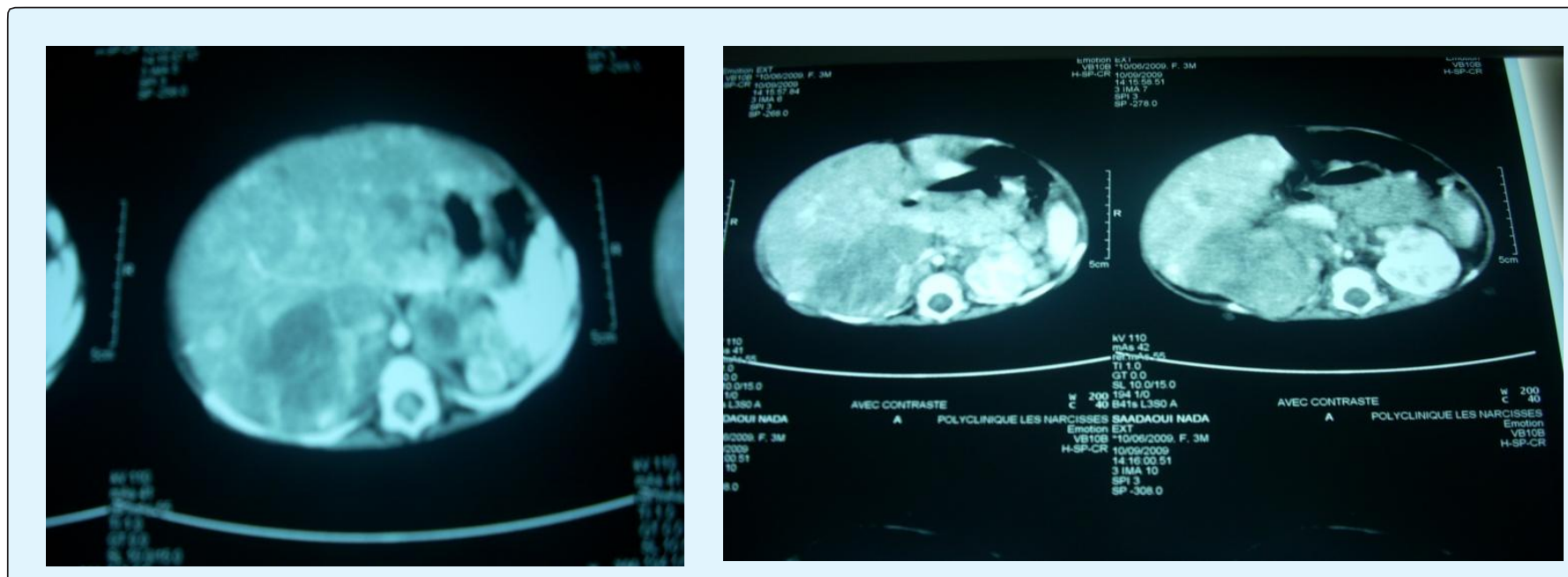

Figure 2: Abdominal CT scan.

The patient underwent a resection of the right adrenal mass, and biopsy of left adrenal mass and liver. The result with pathologic immune histochemical study concluded for a right adrenal neuroblastoma poorly differentiated and invasive high-grade malignancy with liver and controlateral location. Research NMYC amplification has not been available nor any other markers of the tumor.
The diagnosis of bilateral neuroblastoma with liver metastasis associated with Cushing's syndrome was retained and the child was put under antihypertensive therapy and ultrasound monitoring. The short-term trend showed a decline in blood pressure with decreased volume of subcutaneous nodules and regression of Cushing's syndrome including disappearance of telangiectasias, decreased facial 


\section{Open Access Journal of Cancer \& Oncology}

puffiness and weight (weight 7K200 after 1month) (Figure 3).

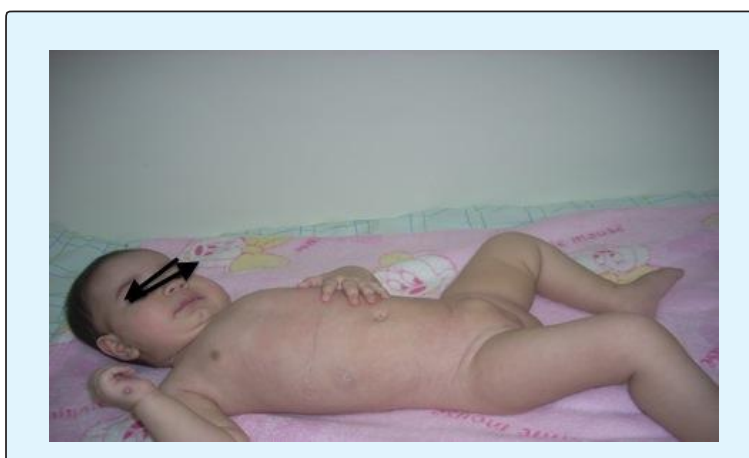

Figure 3: 1 Month After Tumor Resection.

At 9 months of age, Nada presented a reappearance of new nodules in the neck and back, but without any others signs. At 11 months of age, she presented seizures in the right side and coma. The Clinical examination showed no sensory, nor motor response, seizures at the right side of the body, with recurrence of cutaneous nodules.

A Cerebral CT scan and cerebral MRI were done and showed many lesions with hydrocephalus. The decision was made to put the patient under chemotherapy (cyclophosphamide and vincristine) with valproate de sodium.

But finally she died before starting chemotherapy during a week.

- We didn't think to exclude pheoromocytoma because of positivity of biopsy and age of patient

- Possibility of carrying some special mutations was mentioned but at that time we didn't have a cytogenetic lab in our centre

\section{Discussion}

Bilateral adrenal neuroblastoma is usually diagnosed by imaging. It represents up to $10 \%$ of stage IVs neuroblastoma [1]. It has been reported that neuroblastoma may be responsible for ectopic secretion of adrenocorticotrophin hormone ACTH and also DHA. Ectopic secretion of ACTH is rarely due to neuroblastoma, only 9 cases are described in our knowledge and all were in adult's cases, with tumor location at the ethmoid sinus or hypothalamus [2-8]. Immunohistochemistry is important for the diagnosis of neuroblastoma and to determine the tumor origin of ectopic ACTH secretion [2].

The prognosis of neuroblastoma with bilateral adrenal involvement known as Pepper syndrome is favourable. It occurs during the first year of life, presents without bone metastases and MYC-N amplification [1]. The treatment of the reported cases of neuroblastoma with bilateral adrenal Pepper syndrome rarely includes surgery with bilateral adrenalectomy because of the morbidity caused by adrenal insufficiency, and chemotherapy with or without radiotherapy [1].

It was noted disappearance of signs of Cushing's syndrome and then decrease with normalization of plasma ACTH and cortisol following the tumor resection [2]. In our patient, the search for tumor markers of ACTH was not made but, Cushing's syndrome has clearly regressed after tumor resection. The association of the brain metastasis at the time of diagnosis in all published series remained rare. So information regarding pattern of spread, management, and prognosis is limited. The incidence is at $5 \%$ in the St. Jude's Children's Research Hospital (8 patients with recurrent CNS NB among 160 patients). All cases occurred in children who were treated with low dose chemotherapy. Similar incidences from the same area were quoted from the Institute GustaveRoussy series (7 of 258 patients; 3\%) and from the European Neuroblastoma Study Group (10 of 950 patients; $1 \%$.) Brain metastasis at the time of diagnosis in all published series is rare. The median time to CNS recurrence from the time of initial diagnosis ranges from 13 to 24 months.

Risk factors associated with subsequent development of CNS NB are noted in some series, such as cranial and orbital metastases at the time of diagnosis. No published data exist for the association of other markers of aggressive NB like MYCN amplification, high serum LDH, unfavourable histology, and high serum ferrittin and the CNS metastases. Only Kramer found elevated serum LDH $(1500 \mathrm{U} / \mathrm{mL})$ a significant risk factor [9].

Treatment options in CNS neuroblastoma varies from palliative to aggressive therapies. Surgery and radiotherapy rarely leaded to survival beyond a few months. When chemotherapy was given in addition, etoposide with ifosfamide, cisplatin, or carboplatin were used. The median survival is however only 1 to 5 months and, at best, 14 months $[9,10]$. 


\section{Open Access Journal of Cancer \& Oncology}

\section{Conclusion}

The CNS NB is associated with diagnostic lumbar punctures in patients with known bone marrow disease. It is unclear how to follow up and what treatments could prolong survival.

\section{References}

1. Kerdudo C, CorradiniN , Michon J, Levergera G (2004) Neuroblastome surrénalien bilatéral et syndrome de Pepper à propos de quatre observations4S neuroblastoma with bilateral adrenal tumors. Arch Péd 11(12): 1450-1456.

2. Josephs L, Jones L, Marenette L, Mckeever P (2008) Cushing's Syndrome: An Unusual Presentation of Olfactory Neuroblastoma. Skull Base 18(1): 73-76.

3. Reznik M, Melon J, Lambricht M, Kaschten B, Beckers A (1987) Neuroendocrine tumor of the nasal cavity (esthesioneuroblastoma). Apropos of a case with paraneoplastic Cushing's syndrome. Ann Pathol 7(2): 137-142.

4. Arnesen MA, Scheithauer BW, Freeman S (1994) Cushing's syndrome secondary to olfactory neuroblastoma. Ultrastruct Pathol 18(1-2): 61-68.
5. Yu J, Koch CA, Patsalides A, Chang R, Altemus RM, et al. (2004) Ectopic cushing's syndrome caused by an esthesioneuroblastoma. Endocr Pract 10(2): 119-124.

6. Kanno K, Morokuma Y, Tateno T, Hirono Y, Taki K, et al (2005) Olfactory neuroblastoma causing ectopic ACTH syndrome. Endocr J 52(6): 675-681.

7. Koo BK, An JH, Jeon KH, Choi SH, Cho YM, et al. (2008) Two cases of ectopic adrenocorticotropic hormone syndrome with olfactory neuroblastoma and literature review. Endocr J 55(3): 469-475.

8. Mintzer DM, Zheng S, Nagamine M, Newman J, Benito M (2010) Esthesioneuroblastoma (Olfactory Neuroblastoma) with Ectopic ACTH Syndrome: a multidisciplinary case presentation from the Joan Karnell cancer center of Pennsylvania Hospital, Oncologist 15(1): 51-58.

9. Kramer K, Kushner B, Heller G, Cheung NK (2001) Neuroblastoma Metastatic to the Central Nervous System: The Memorial Sloan-Kettering Cancer Center Experience and A Literature Review. Cancer 91(8): 1510-1519.

10. Paulino AC, Nguyen TX, Barker JL (2003) Brain metastasis in children with sarcoma, neuroblastoma, and Wilm's tumor. Int J Radiat Oncol Biol Phys 57(1): 177-183. 